\title{
Distributed representation and estimation of WFST-based n-gram models
}

\author{
Cyril Allauzen, Michael Riley and Brian Roark \\ Google, Inc. \\ \{allauzen,riley,roark\}@google.com
}

\begin{abstract}
We present methods for partitioning a weighted finite-state transducer (WFST) representation of an n-gram language model into multiple blocks or shards, each of which is a stand-alone WFST n-gram model in its own right, allowing processing with existing algorithms. After independent estimation, including normalization, smoothing and pruning on each shard, the shards can be reassembled into a single WFST that is identical to the model that would have resulted from estimation without sharding. We then present an approach that uses data partitions in conjunction with WFST sharding to estimate models on orders-of-magnitude more data than would have otherwise been feasible with a single process. We present some numbers on shard characteristics when large models are trained from a very large data set. Functionality to support distributed n-gram modeling has been added to the open-source OpenGrm library.
\end{abstract}

\section{Introduction}

Training n-gram language models on ever increasing amounts of text continues to yield large model improvements for tasks as diverse as machine translation (MT), automatic speech recognition (ASR) and mobile text entry. One approach to scaling n-gram model estimation to peta-byte scale data sources and beyond, is to distribute the storage, processing and serving of n-grams (Heafield, 2011). In some scenarios - most notably ASR - a very common approach is to heavily prune models trained on large resources, and then pre-compose the resulting model off-line with other models (e.g., a pronunciation lexicon) in order to optimize the model for use at time of firstpass decoding (Mohri et al., 2002). Among other things, this approach can impact the choice of smoothing for the first-pass model (Chelba et al., 2010), and the resulting model is generally stored as a weighted finite-state transducer (WFST) in order to take advantage of known operations such as determinization, minimization and weight pushing (Allauzen et al., 2007; Allauzen et al., 2009; Allauzen and Riley, 2013). Even though the resulting model in such scenarios is generally of modest size, there is a benefit to training on very large samples, since model pruning generally aims to minimize the KL divergence from the unpruned model (Stolcke, 1998).

Storing such a large n-gram model in a single WFST prior to model pruning is not feasible in many situations. For example, speech recognition first pass models may be trained as a mixture of models from many domains, each of which are trained on billions or tens of billions of sentences (Sak et al., 2013). Even with modest count thresholding, the size of such models before entropybased pruning would be on the order of tens of billions of n-grams.

Storing this model in the WFST n-gram format of the OpenGrm library (Roark et al., 2012) allocates an arc for every n-gram (other than end-ofstring n-grams) and a state for every n-gram prefix. Even using very efficient specialized n-gram representations (Sorensen and Allauzen, 2011), a single FST representing this model would require on the order of 400GB of storage, making it difficult to access and process on a single processor.

In this paper, we present methods for the distributed representation and processing of large WFST-based n-gram language models by partitioning them into multiple blocks or shards. Our sharding approach meets two key desiderata: 1) each sub-model shard is a stand-alone "canonical format" WFST-based model in its own right, providing correct probabilities for a particular subset of the n-grams from the full model; and 2) once ngram counts have been sharded, downstream pro- 


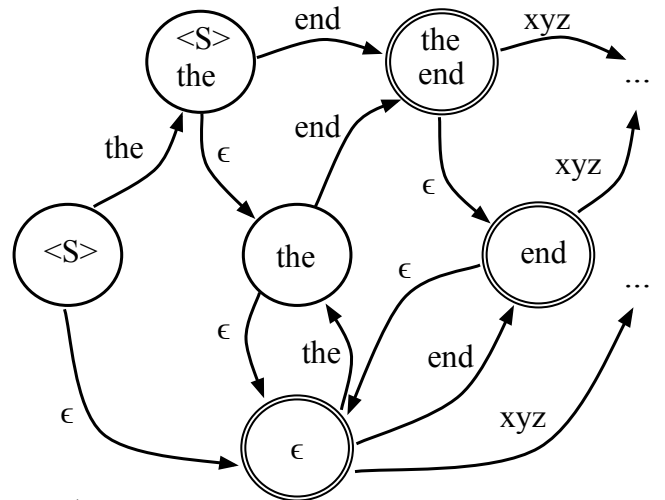

Figure 1: Schematic of canonical WFST n-gram format, unweighted for simplicity. Each state shows the history it encodes for convenience (they are actually unlabeled). Final states are denoted with double circle.

cessing such as model normalization, smoothing and pruning, can occur on each shard independently. Methods, utilities and convenience scripts have been added to the OpenGrm NGram library ${ }^{1}$ to permit distributed processing. In addition to presenting design principles and algorithms in this paper, we will also outline the relevant library functionality.

\section{Canonical WFST n-gram format}

We take as our starting point the standard 'canonical' WFST n-gram model format from OpenGrm, which is presented in Roark et al. (2012) and at ngram.opengrm.org, but which we summarize briefly here. Standard n-gram language models can be presented in the following well-known backoff formulation:

$\mathrm{P}(w \mid h)= \begin{cases}\hat{\mathrm{P}}(w \mid h) & \text { if } c(h w)>0 \\ \alpha(h) \mathrm{P}\left(w \mid h^{\prime}\right) & \text { otherwise }\end{cases}$

where $w$ is the word (or symbol) being predicted based on the previous history $h$, and $h^{\prime}$ is the longest proper suffix of $h$ (or $\epsilon$ if $h$ is a single word/symbol). The backoff weight $\alpha(h)$ ensures that this is a proper probability distribution over symbols in the vocabulary, and is easily calculated based on the estimates $\hat{\mathrm{P}}$ for observed n-grams. Note that interpolated n-gram models also fit this formulation, if pre-interpolated.

Figure 1 presents a schematic of the WFST ngram model format that we describe here. The WFST format represents n-gram histories $h$ as states $^{2}$, and words $w$ following $h$ as arcs leaving

\footnotetext{
${ }^{1}$ ngram.opengrm.org

${ }^{2}$ For convenience, we will refer to states as encoding (or representing) a history $h$ - or even just call the state $h-$ though there is no labeling of states, just arcs.
}

the state that encodes $h$. There is exactly one unigram state (labeled with $\epsilon$ in Figure 1), which represents the empty history. For every state $h$ in the model other than the unigram state, there is a special backoff arc, labeled with $\epsilon$, with destination state $h^{\prime}$, the backoff state of $h$. For an n-gram $h w$, the arc labeled with $w$ leaving history state $h$ will have as destination the state $h w$ if $h w$ is a proper prefix of another n-gram in the model; otherwise the destination will be $h^{\prime} w$. The start state of the model WFST represents the start-of-string history (typically denoted $<\mathrm{S}>$ ), and the end-ofstring $(</ \mathrm{S}>)$ probability is encoded in state final costs. Neither of these symbols labels any arcs in the model, hence they are not required to be part of the explicit vocabulary of the model. Costs in the model are generally represented as negative log counts or probabilities, and the backoff arc cost from state $h$ is $-\log \alpha(h)$.

With the exception of the start and unigram states, every state $h$ in the model is the destination state of an $n$-gram transition originating from a prefix history, which we will term an 'ascending' n-gram transition. If $h=w_{1} \ldots w_{k}$ is a state in the model $(k>0$ and if $k=1$ then $w_{1} \neq<\mathrm{S}>$ ), then there also exists a state in the model $\bar{h}=w_{1} \ldots w_{k-1}$ and a transition from $\bar{h}$ to $h$ labeled with $w_{k}$. We will call a sequence of such ascending n-gram transitions an ascending path, and every state in the model (other than unigram and start) can be reached via a single ascending path from either the unigram state or the start state. This plus the backoff arcs make the model fully connected.

\section{Sharding count n-gram WFSTs}

\subsection{Model partitioning}

Our principal interest in breaking (or sharding) this WFST representation into smaller parts lies in enabling model estimation for very large training sets by allowing each shard to be processed (normalized, smoothed and pruned) as independently as possible. Further, we would like to simply use existing algorithms for each of these stages on the model shards. To that end, all of the arcs leaving a particular state must be included in the same shard, hence our sharding function is for states in the automaton, and arcs go with their state of origin. We shard the $n$-gram WFST model into a collection of $\mathrm{n}$-gram WFSTs by partitioning the histories into intervals on a colexicographic ordering defined below. The model's symbol table maps from sym- 
bols in the model to unique indices that label the arcs in the WFST. We use indices from this symbol table to define a total order $<_{V}$ on our vocabulary augmented with start-of-string token which is assigned index $0 .{ }^{3}$ We then define the colexicographic (or reverse lexicographic) order $<$ over $V^{*}$ recursively on the length of the sequences as follow. For all $x, y \neq \epsilon$, we have $\epsilon<x$ and

$$
x<y \text { iff }\left\{\begin{array}{l}
x_{|x|}<_{V} y_{|y|} \text { or, } \\
x_{|x|}=y_{|y|} \text { and } \bar{x}<\bar{y}
\end{array}\right.
$$

where $\bar{x}$ denotes the longest prefix of $x$ distinct from $x$ itself. The colexicographic interval $[x, y)$ then denotes the set of sequences $z$ such that $x \leq$ $z<y$.

For example, assuming symbol indices the $=1$ and end $=2$, the colexicographic ordering of the states in Figure 1 is:

\begin{tabular}{crr} 
Colex. & \multicolumn{2}{c}{ State histories } \\
Order & (as words) & (as indices) \\
0 & $\epsilon$ & $\epsilon$ \\
1 & $<\mathrm{S}>$ & 0 \\
2 & the & 1 \\
3 & $<\mathrm{S}>$ the & 01 \\
4 & end & 2 \\
5 & the end & 12
\end{tabular}

If we want, say, 4 shards of this model (at least, the visible part in the schematic in Figure 1), we can partition the state histories in 4 intervals; for example:

$$
\begin{aligned}
& \{[\epsilon, 1),[1,2),[2,12),[12,3)\}= \\
& \{\{\epsilon, 0\},\{1,01\},\{2\},\{12\}\} .
\end{aligned}
$$

By convention, we write the interval $[x, y)$ as $x_{1} \ldots x_{l}: y_{1} \ldots y_{m}$. Thus, the above partition would be written as: ${ }^{4}$

$$
\begin{array}{rlll} 
& 0 & : & 1 \\
1 & : & 2 \\
& 2 & : & 1 \\
& 2 & 2 \\
1 & 2 & : & 3
\end{array}
$$

While this partitions the states into subsets, it remains to turn these subsets into stand-alone, connected WFSTs with the correct topology to allow for direct use of existing language model estimation algorithms on each shard independently. For this to be the case, we need to: (1) be

\footnotetext{
${ }^{3}$ Not to be confused with the convention that $\epsilon$ has index 0 in FST symbol tables.

${ }^{4}$ We omit the empty history $\epsilon$ from the interval specification since it is always assigned to the first interval.
}

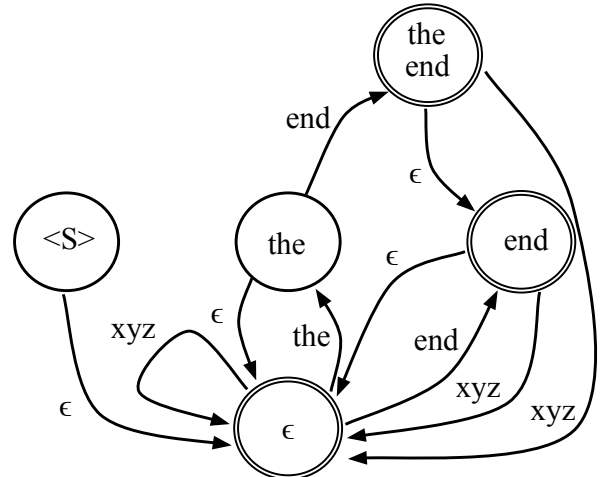

Figure 2: Schematic of a completed shard of the model in Figure 1. The state corresponding to the history 'the end' is the only state strictly in-context for this shard.

able to reach each state via the correct ascending path from the start or unigram state, with correct counts/probabilities; (2) have backoff states of all in-shard states, along with their arcs, for calculating backoff costs; and (3) correctly assign all arc destinations within each new WFST.

\subsection{Model completion}

Given a set of states to include in a context shard, the shard model must be 'completed' to include all of the requisite states and arcs needed to conform to the canonical n-gram topology. We step through each of the key requirements in turn. We refer to those states that fall within the context interval as 'strictly in-context'. Figure 2 shows a schematic of the shard model that results for the context 12 : 3 , which we will refer to when illustrating particular requirements. Only the state corresponding to 'the end' is strictly in-context for this particular shard. All states that are suffixes of strictly in-context states are also referred to as in-context (though not strictly so), since they are needed for proper normalization - i.e., calculation of $\alpha(h)$ in the recursive $\mathrm{n}$-gram model definition in equation 1 . Hence, the state corresponding to 'end' in Figure 2 is in-context and is included in the shard, as is the unigram state.

The start state and all states and transitions on ascending paths from the start and unigram states to in-context states must be included, so that states that are in-context can be reached from the start state. Thus, the state corresponding to 'the' in Figure 2 must be included, along with its arc labeled with 'end', since they are on the ascending path to 'the end', which is strictly in-context.

For every state in the model, the backoff arc should allow transition to the correct backoff state. Finally, for all arcs (labeled with $w$ ) leaving states $h$ that have been included in the shard model, their 
destination must be the longest suffix of $h w$ that has been included as a state in the shard model. The arcs labeled with 'xyz' in Figure 2 all point to the unigram state, since no states representing histories ending in 'xyz' are in the shard model.

For the small schematic example in Figures 1 and 2, there is not much savings from sharding after completing the shard model: only one state and four arcs from the observed part of the model in Figure 1 were omitted in the schematic in Figure 2. And it is clear from the construction that there will be some redundancy between shards in the states and arcs included when the shard model is completed. But for large models, each shard will be a small fraction of the total model. Note that there is a tradeoff between the number of shards and the amount of redundancy across shards.

Another way to view the shard model in Figure 2 relative to the full model in Figure 1 is as a pruned model, where the arcs and states that were pruned are precisely those that are not needed within that particular shard. This perspective is useful when discussing distributed training in the next section.

\section{Distributed training of n-gram models}

When presenting model sharding in the previous section, we had access to the specific states in the model schematic, and defined the contexts accordingly. When training a model from data at the scale that requires distributed processing, the full model does not exist to inspect and partition. Instead, we must derive the context sharding in some fashion prior to training the model. We will thus break this section into two parts: first, deriving context intervals for model sharding; then estimating models given context intervals.

\subsection{Deriving context intervals}

Given a large corpus, there are a couple of ways to approach efficient calculation of effective context intervals. Effective in this case is balanced, i.e., one would like each sharded model to be of roughly the same size, so that the time for model estimation is roughly commensurate across shards and lagging shards are avoided.

The first approach is to build a smaller footprint model than the desired model, which would take a fraction of the time to train, then derive the contexts from that model. For example, if one wanted to train a 5-gram model from a billion word corpus, then one may derive context intervals based on trigram model trained by sampling one out of every hundred sentences from the corpus. Given that more compact model, it is relatively straightforward to examine the storage required for each state and choose a balanced partition accordingly. At higher orders and with the full sample, the size of each shard may ultimately differ, but we have found that estimating relative shard sizes based on lower-order sampled models is effective at providing functional context intervals. See section 5 for specific OpenGrm NGram library functionality related to context interval estimation.

Another method for deriving context intervals is to accumulate the set of n-grams into a large collection, sort it by history in the same colexicographic order as is used to define the context intervals, and then take quantiles from that sorted collection. This can lead to more balanced shards than the previous method, though efficient methods for distributed quantile extraction from collections of that sort is beyond the scope of this paper.

\subsection{Estimating models given context intervals}

Given a definition of $k$ context intervals $C_{1} \ldots C_{k}$, we can train sharded models on very large data sets as follows:

1. Partition data into $m$ data shards $D_{1} \ldots D_{m}$

2. For each data shard $D_{i}$

(a) Count the n-grams from $D_{i}$ and build full WFST n-gram representation $T_{i}$

(b) Split $T_{i}$ into $k$ shard models $T_{i 1} \ldots T_{i k}$

3. For each context interval $C_{j}$, merge counts $T_{\cdot j}$ from all data shards: $F_{j}=\operatorname{Merge}_{i=1}^{m}\left(T_{i j}\right)$

4. Perform these global operations on collection $F_{1} \ldots F_{k}$ to prepare for model estimation:

(a) Transfer correct counts as needed across shards (see Section 4.2.4 below).

(b) Derive resources such as count-ofcounts by aggregating across shards.

5. Normalize, smooth, prune each $F_{j}$ as needed: $M_{j}=\operatorname{MakeModel}\left(F_{j}\right)$

6. Merge model shards: $M=\operatorname{Merge}_{j=1}^{k}\left(M_{j}\right)$

We now go through each of these 6 stages one by one in the following sub-sections.

\subsubsection{Partition data}

Given a large text corpus, this simply involves placing each string into one of $m$ separate collections, preferably of roughly equal size. 


\subsubsection{Count and split data shards}

For each data shard $D_{i}$, perform n-gram counting exactly as one would in a non-distributed scenario. (See Section 5 for specific commands within the OpenGrm NGram library.) This results in an ngram count WFST $T_{i}$ for each data shard. Using the context interval specifications $C_{1} \ldots C_{k}$ we then split $T_{i}$ into $k$ shard models. Because we have the full model $T_{i}$, we can determine exactly which states and arcs need to be preserved for each context interval, and prune the rest away.

\subsubsection{Merge sharded models}

For each context interval $C_{j}$, there will be a shard model $T_{i j}$ for every data shard $D_{i}$. Standard count merging will yield the correct counts for all in-context n-grams and the correct overall model topology, i.e., every state and arc that is required will be there. However, n-grams that are not incontext may not have the correct count, since they may have occurred in a data shard but were not included in the context shard due to the absence of any in-context n-grams for which it is a prefix.

To illustrate this point, consider a scenario with just two data shards, $D_{1}$ and $D_{2}$, and a context shard $C_{j}$ that only includes the n-gram history 'foo bar baz' strictly in-context. Suppose 'foo bar' occurs 10 times in $D_{1}$ and also 10 times in $D_{2}$, while 'foo bar baz' occurs 3 times in $D_{1}$ but doesn't occur at all in $D_{2}$. Recall that states and ascending arcs that are not in-context are only included in the shard model as required to ascend to the incontext states. In the absence of 'foo bar baz' in $T_{2 j}$, the n-gram arc and state corresponding to 'foo bar' will not be included in that shard, despite having occurred 10 times in $D_{2}$. When the counts in $T_{1 j}$ and $T_{2 j}$ are merged, 'foo bar' will be included in the merger, but will only have counts coming from $T_{1 j}$. Hence, rather than the correct count of 20 , that n-gram will just have a count of 10 . The correct count of 'foo bar' is only guaranteed to be found in the shard for which it is in-context.

To get the correct counts in every shard that needs them, we must perform a transfer operation to pass correct counts from shards where ngrams are strictly in-context to shards where they are needed as prefixes of other n-grams.

\subsubsection{Global operations on the collection}

Transfer: As mentioned above, count merging of sharded count WFSTs across data shards yields correct counts for in-context states, as well as the correct WFST topology - i.e., all needed n-grams are included - but is not guaranteed to have the correct counts of n-grams that are not in-context. For each shard $F_{i}$, however, we know which ngrams we need to get the correct count for, and can easily calculate the context shard that these ngrams fall into. Using that information, a transfer of correct counts is effected via the following three stages:

1. For each shard $F_{i}$, for each $F_{j}(j \neq i)$, prune $F_{i}$ to only those n-grams that are strictly incontext for context $C_{j}$, and send the resulting $F_{i j}$ to shard $F_{j}$ to give correct counts.

2. For every shard $F_{j}$, provide correct counts for each incoming $F_{. j}$ requiring them and return to the appropriate shard $F_{i}$.

3. For every shard $F_{i}$, update counts from incoming $F_{i}$.

Only needed n-grams are processed in this transfer algorithm, which we will term the "standard" transfer algorithm in the experimental results. Let $Q_{i}$ be the set of states for shard $F_{i}$. Each state is an n-gram of length less than $n$ (where $n$ is the order of the model) that must have its correct count requested from the shard where it is strictly in context. This leads to a complexity of $O\left(n \sum_{i=1}^{k}\left|Q_{i}\right|\right)$.

An alternative, which we will term the "byorder" transfer algorithm, performs transfer of a more restricted set of $\mathrm{n}$-grams in multiple phases, which occur in ascending n-gram order. Note that, when transfer of correct counts for a particular ngram is requested, the correct counts for all prefixes of that n-gram can also be collected at the same time at no extra cost, provided the prefix counts are correct in the shard where we request them, even though the prefixes may or may not be in-context. By processing in ascending $n$-gram order, we can guarantee that the prefixes of requested n-grams have already been updated to the correct counts. If we can update the counts of n-gram prefixes, we can defer the transfer of an out-ofcontext n-gram's count until an update is required. The correct count of an out-of-context n-gram of order $n$ is thus only requested if one of the following two conditions hold: (1) its count may be requested by another context shard from the current context shard during the transfer phase of order $n+1$; or (2) its count would not be transferred at some order greater than $n$, hence must be transferred now to be correct at the end of transfer. The former condition holds if the n-gram arc has an origin state that is out-of-context and a destination 
state that is strictly in-context. The latter condition holds if the n-gram arc's origin state is out-ofcontext, its destination state is in-context (though not strictly in-context), and the n-gram is not a prefix of any in-context state. We will call an n-gram of order $n$ that meets either of those conditions "needed at order $n$ ". Then, for each order $n$ from 1 to the highest order in the model, transfer is carried out by replacing step number 1 in the standard transfer algorithm above with the following:

1. For each shard $F_{i}$, for each $F_{j}(j \neq i)$, prune $F_{i}$ to only those n-grams that are strictly incontext for context $C_{j}$, and are needed at order $n$, along with all prefixes of such n-grams. If the resulting $F_{i j}$ is non-empty, send it to shard $F_{j}$ to give correct counts.

The rest of transfer at order $n$ proceeds as before. In this algorithm, a shard requests an n-gram only if the destination state of its corresponding n-gram arc is in-context. This leads to a complexity in $O\left(n \sum_{i=1}^{k}\left|Q_{i}^{c}\right|\right)$ where $Q_{i}^{c}$ denotes the set of states in shard $F_{i}$ corresponding to in-context histories for that shard. This is a complexity reduction from the standard transfer algorithm above, since $\left|Q_{i}\right| / n<\left|Q_{i}^{c}\right|<\left|Q_{i}\right|$.

Counts-of-counts: Deriving counts-of-count histograms is key for certain smoothing methods such as Katz (1987). Each shard $F_{i}$ can produce a histogram from only those n-grams that are strictly in-context, then the histograms can be aggregated straightforwardly across shards to produce a global histogram, since each n-gram is strictly in-context in only one shard.

\subsubsection{Process count shards}

Given the correct counts in each of the count shards $F_{i}$, we can proceed to use existing, standard n-gram processing algorithms to normalize, smooth and prune each of the models independently. These algorithms are linear in the size of the model being processed. With some minor exceptions, existing WFST-based language modeling algorithms, such as those in the OpenGrm NGram library, can be applied to each shard independently. We mention two such exceptions in turn, both impacting the correct application of model pruning algorithms after the model shard has been normalized and smoothed.

First, whereas common smoothing algorithms such as Katz (1987) and absolute discounting (Ney et al., 1994) will properly discount and normalize all n-grams in the model shard, WittenBell smoothing (Witten and Bell, 1991) will yield correct smoothed probabilities for in-context ngrams, but for n-grams not in-context in the current shard, the smoothed probabilities will not be guaranteed to be correctly estimated. This is because Witten-Bell smoothing is defined in terms of the number of words that have been observed following a particular history, which in the WFST encoding of the n-gram model is represented by the number of arcs (other than the backoff arc) leaving the history state (plus one if the state is final). While for any in-context state $h$, all of the arcs leaving the state will be present, some of the other n-gram states that were included to create the model topology - notably the states along the ascending path to in-context states - will not typically have all of the arcs that they have in their own shard. Hence the denominator in Witten-Bell smoothing (the count of the state plus the number of words observed following the history) cannot be calculated locally, and the direct application of the algorithm will end up with mis-estimated n-gram probabilities along the ascending paths.

If no pruning is done, then only the in-context probabilities matter, and merging can take place with no issues (see the next section 4.2.6).

Pruning algorithms, however, such as relative entropy pruning (Stolcke, 1998), typically use the joint n-gram probability - $\mathrm{P}(h w)$ - when calculating the scores that are used to decide whether to prune the n-gram or not. This joint probability is calculated by taking the product of all ascending path conditional probabilities. If the ascending path probabilities are wrong, these scores will also be wrong, and pruning will proceed in error. For Katz and absolute discounting, the ascending probabilities are correct when calculated on the shard independently of the other shards (when given counts-of-counts); but Witten Bell will not be immediately ready for pruning.

To get correct pruning for a sharded WittenBell model, another round of the transfer algorithm outlined in Section 4.2.4 is required, to retrieve the correct probabilities of ascending arcs in each shard.

The second issue to note here arises when pruning the model to have a particular number of desired n-grams in the model. For example, in some of the trials that we run in Section 6 we prune the $\mathrm{n}$-gram models to result in 100 million $\mathrm{n}$-grams in the final model. To establish a pruning threshold that will result in a given total number of $n$-grams across all shards, the shrinking score must be cal- 
culated for every n-gram in the collection and then these scores sorted to derive the right threshold. This requires a process not unlike the counts-ofcounts aggregation presented in Section 4.2.4, yet with a sorting of the collection rather than compilation into a histogram.

Once all of the model shards have been normalized, smoothed and pruned using standard WFSTbased n-gram algorithms, the shards can be reassembled to produce a single WFST.

\subsubsection{Merge model shards}

Merging the shard models into a single WFST ngram model is a straightforward special case of general model merging, whereby two models are merged into one. In general, model merging algorithms of two WFST models with canonical ngram topology will: (a) result in a new model with canonical n-gram topology; and (b) the n-gram costs in the new model are some function of the $\mathrm{n}$-gram costs in the two models. If the models are being linearly interpolated, then the n-gram probability will be calculated as $\lambda p_{1}+(1-\lambda) p_{2}$, where $p_{k}$ comes from the $k$ th model, and the $\mathrm{n}$-gram cost will be the negative log of that probability. ${ }^{5}$

To merge model shards $M_{1}$ and $M_{2}$, we must know, for each state $h$, whether $h$ is in-context for $M_{1}$ or $M_{2}$. The n-gram cost in the merged model is $c_{2}$ if $h$ is in-context for $M_{2}$; and $c_{1}$ otherwise, where $c_{k}$ is the cost of the n-gram in $M_{k}$. If we start with an arbitrary model shard and designate that as $M_{1}$, then we can merge each other shard into the merged model in turn, and designate the resulting merged model as $M_{1}$ for a subsequent merge. By the end of merging in every context, all of the $n$-grams in the final model will have been merged in, so they will all have received their correct probabilities. The resulting WFST will have the same probabilities as it would if the model had been trained in a single process.

\section{OpenGrm distributed functionality}

While most of these distributed functions will likely be implemented in some kind of large, dataparallel processing system ${ }^{6}$, such as MapReduce (Dean and Ghemawat, 2008), these pipelines will rely upon core OpenGrm NGram library functions to count, make, prune and merge models. The

\footnotetext{
${ }^{5}$ Backoff arc costs can then be calculated in closed form.

${ }^{6} \mathrm{We}$ have implemented an end-to-end pipeline, which makes use of the OpenGrm NGram library, in Flume (Chambers et al., 2010). Results in Section 6 were generated with this pipeline.
}

OpenGrm NGram library now includes some distributed functionality, along with a convenience script to illustrate the sort of approach we have described in this paper.

Recall that the basic approach involves sharding the data, counting $\mathrm{n}$-grams on each data shard separately, and then splitting the counts from each data shard into context shards. Two commandline utilities in OpenGrm provide functionality for (1) defining context shards; and (2) splitting an ngram WFST based on given context shards. One method described in Section 4.1 for deriving context shards is to train a smaller model (e.g., lower order and/or sampled from the full target training scenario) and then derive balanced context shards from that smaller model. For example, if we want to train a 5-gram model on 1B words of text, we might count ${ }^{7}$ trigrams from every 100th sentence, yielding the n-gram count WFST $3 g$.fst. Then the command line binary ngramcontext can make use of the sampled counts to derive a balanced sharding of the requested size:

ngramcontext -- contexts $=10 \mathrm{3g}$.fst >ctx.txt

The resulting text file (ctx.txt) will look something like this:

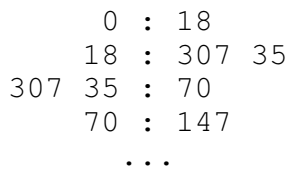

as discussed in Section 3.1. Given these context definitions, we can now use ngramsplit to partition full count WFSTs derived from particular data shards. For example, suppose that we counted 5-grams from data shard $k$, yielding DS-k. $5 \mathrm{~g}$-counts. fst. Then we can produce 10 count shards as follows:

ngramsplit -- contexts $=$ ctx.txt -- complete DS-k. $5 \mathrm{~g}$-counts.fst DS-k.5g-counts

which would result in 10 count shard WFSTs DS-k.5g-counts.0000i for $0 \leq i<10$. The --complete flag ensures that all required ngrams are included in the shard, not just those strictly in-context. Once this has been done for all data shards, the counts for each context shard can be merged across the data shards, i.e., ngrammerge using the countmerge method on DS-*.5g-counts.0000i for all $i$.

As discussed in Section 4.2.4, once we have the merged counts for each model shard, we must perform a transfer of the correct counts. This involves

\footnotetext{
${ }^{7}$ See Roark et al. (2012) for details on n-gram counting in the OpenGrm library.
} 


\begin{tabular}{|c|c|c|c|c|c|c|c|c|}
\hline \multirow[b]{2}{*}{$\begin{array}{l}\text { Corpus } \\
\text { (words; sents) }\end{array}$} & \multicolumn{3}{|c|}{ n-grams } & \multicolumn{2}{|c|}{ time (hours) to } & \multirow[b]{2}{*}{$\begin{array}{l}\text { model } \\
\text { shards }\end{array}$} & \multirow{2}{*}{$\begin{array}{l}\text { pct. in- } \\
\text { context } \\
\text { ngrams }\end{array}$} & \multirow{2}{*}{$\begin{array}{c}\text { largest to } \\
\text { smallest } \\
\text { shard ratio }\end{array}$} \\
\hline & order & total & $\begin{array}{c}\text { target } \\
\text { per shard }\end{array}$ & $\begin{array}{l}\text { preproc. } \\
+ \text { count }\end{array}$ & $\begin{array}{l}\text { make, } \\
\text { prune + }\end{array}$ & & & \\
\hline \multirow{4}{*}{$\begin{array}{l}\text { Billion word } \\
\text { benchmark (BWB) } \\
(769 \mathrm{M} ; 30.3 \mathrm{M})\end{array}$} & \multirow[t]{2}{*}{3} & \multirow[t]{2}{*}{$238 \mathrm{M}$} & $4 \mathrm{M}$ & 1.5 & 1.2 & 59 & 56.0 & 1.20 \\
\hline & & & $40 \mathrm{M}$ & 1.6 & 1.6 & 5 & 84.8 & 1.07 \\
\hline & \multirow[t]{2}{*}{5} & \multirow[t]{2}{*}{$1.14 \mathrm{~B}$} & $4 \mathrm{M}$ & 4.1 & 2.0 & 285 & 36.8 & 2.07 \\
\hline & & & $40 \mathrm{M}$ & 4.7 & 3.5 & 28 & 50.5 & 1.26 \\
\hline \multirow{2}{*}{$\begin{array}{l}\text { Search queries (SQ) } \\
(70 \mathrm{~B} ; 13.2 \mathrm{~B})\end{array}$} & \multirow[t]{2}{*}{5} & \multirow[t]{2}{*}{$16.6 \mathrm{~B}$} & $4 \mathrm{M}$ & 23.4 & 8.9 & 5090 & 38.8 & 1.93 \\
\hline & & & $40 \mathrm{M}$ & 10.2 & 7.1 & 502 & 64.4 & 1.77 \\
\hline
\end{tabular}

Table 1: Sharding characteristics and time to estimate under different training scenarios. As noted in Section 6, times are not comparable if n-gram order or size of corpus are different, and times should be interpreted as a relatively coarse measure of work. The last two columns (100*in-context/total n-ngrams and the ratio of sizes in ngrams) indicate shard redundancy.

splitting again and using the command line binary ngramtransfer twice: once to extract the correct counts from the correct shards; and once to return the extracted counts to the shards requesting them. We refer the reader to Section 4.2.4 for high level detail, and the convenience script ngram.sh in the OpenGrm NGram library for specifics.

Several new functions have been added via options to existing command line binaries in the OpenGrm NGram library. For example, ngramcount can now produce counts of counts (--method=count_of_counts) and produce them only for a specified context shard. Further, ngrammerge has a context_merge method, which uses a derived class of OpenGrm's NGramMerge class to correctly reassemble count or language model sharded WFSTs into a single WFST. See the script ngram.sh in the OpenGrm NGram library for details.

In the next section, we provide some data on the characteristics of n-gram models of different orders and sizes when they are trained via sharding.

\section{Shard size versus redundancy}

As stated earlier, we use Flume (Chambers et al., 2010) in C++ to distribute our OpenGrm NGram model training. This system is not currently publicly available, but within it we use methods generally very similar to what is available in OpenGrm, just pipelined together in a different way. One difference between the Flume version and ngram.sh is the method for deriving contexts, which in Flume is based on efficient quantiles extracted from the set of n-grams. While this is also a sampling method for deriving the contexts, the ordering constraints of quantiles do often lead to better (though not perfect) estimates of balanced shards. Additionally, the Flume system that was used to generate these numbers uses a smart distributed processing framework, which allocates processors based on estimated size of the process. This impacts the interpretability of timing results, as noted below.

Table 1 presents some characteristics of language model training under several conditions which demonstrate some of the tradeoffs in distributing the model in slightly different ways. From the Billion Word Benchmark (BWB) corpus (Chelba et al., 2014), we train trigram and 5gram language models with different parameterizations for determining the model sharding. We also report results on a proprietary 70 billion word collection of search queries (SQ), also with different sharding parameterizations. For the BWB trials, no symbol or n-gram frequency cutoffs were used, but for the search queries, as part of the preprocessing and counting, we selected the 4 million most common words from the collection to include in the vocabulary (all others mapped to an out-of-vocabulary token) and limited 4-grams and 5-grams to those occurring at least twice or 4 times, respectively. All trigram models were pruned to 50 million n-grams prior to shard merging (reassembling into a single WFST), and 5gram models were pruned to 100 million n-grams. For these trials, the standard transfer algorithm in Section 4.2.4 was used. Run times are averaged over five independent runs.

Note that, due to the smart distributed processing framework, the times are not comparable if ngram order or size of corpus are different. Further, due to distributed processing with resource contention, etc., the times should be interpreted as a coarse measure of work. That said, we note that in the largest scenario, parameterizing for relatively small shards (4M n-grams in-context per shard) yields over 5000 shards, which results in extra time in transfer (hence higher count times) and in final merging of the contexts (hence higher make, 


\begin{tabular}{|c|c|c|c|c|c|}
\hline & target & trans & time $(1$ & ours) to & ount \\
\hline Task & $\begin{array}{l}\text { per } \\
\text { shard }\end{array}$ & $\begin{array}{l}\text { by } \\
\text { order }\end{array}$ & $\begin{array}{l}\text { before } \\
\text { trans. }\end{array}$ & $\begin{array}{l}\text { trans. } \\
\text { to end }\end{array}$ & total \\
\hline \multirow[t]{4}{*}{ BWB } & \multirow[t]{2}{*}{$4 \mathrm{M}$} & $\mathrm{N}$ & 2.4 & 1.7 & 4.1 \\
\hline & & $\bar{Y}$ & 2.1 & 3.5 & 5.5 \\
\hline & \multirow[t]{2}{*}{$40 \mathrm{M}$} & $\mathrm{N}$ & 2.6 & 2.1 & 4.7 \\
\hline & & $\mathrm{Y}$ & 3.1 & 5.9 & 9.0 \\
\hline \multirow[t]{4}{*}{ SQ } & \multirow[t]{2}{*}{$4 \mathrm{M}$} & $\mathrm{N}$ & 4.7 & 18.7 & 23.4 \\
\hline & & $\mathrm{Y}$ & 4.5 & 7.6 & 12.1 \\
\hline & \multirow[t]{2}{*}{$40 \mathrm{M}$} & $\mathrm{N}$ & 5.7 & 4.4 & 10.2 \\
\hline & & $\bar{v}$ & 5.9 & 6.7 & 12.6 \\
\hline
\end{tabular}

Table 2: Counting time broken down between stages occurring before transfer and those occurring from transfer to the end of counting, using either the original transfer algorithm or transferring by order.

prune, etc. times). With larger shard sizes (and hence fewer shards), the percentage of n-grams in each shard that are in-context (rather than ascending or backoff $n$-grams) is higher, and the size of the largest shard (in terms of total n-grams in the shard, both in-context and not) is much closer in size to the smallest shard, leading to better load balancing. Smaller shards, however, will generally distribute more effectively for many of the estimation tasks, leading to some speedups relative to fewer, larger shards.

Table 2 presents counting times for the 5-gram trials using both the standard transfer algorithm reported in Table 1 and the alternate "by order" transfer algorithm outlined in Section 4.2.4. The times are broken down into the part of counting before transfer and the part including transfer until the end. From these we can see that in scenarios with a very large number of shards - e.g., SQ with $4 \mathrm{M}$ target per shard, which yields more than 5000 shards - the "by order" transfer algorithm is much faster than the standard algorithm, leading to a factor of 2 speedup overall. However, increasing the target number of $n$-grams per shard, thus yielding fewer shards, is overall a more effective way to speedup processing. For much larger training scenarios, when even $40 \mathrm{M}$ n-grams per shard would yield a large number of shards, one would expect this alternative transfer algorithm to be useful. Otherwise, the additional overhead of the additional stages simply adds to the processing time.

\section{Related work}

Brants et al. (2007) presented work on distributed language model training that has been very influential. In that work, n-grams were sharded based on a hash function of the first words of the n-gram, so that prefix n-grams, which carry normalization counts, end up in the same shard as those requiring the normalization. Because suffix n-grams do not end up in the same shard, smoothing methods that need access to backoff histories, such as Katz, require additional processing.

In contrast, our sharding is on the suffix of the history, which ensures that all n-grams with the same history fall together, and very often the backoff histories also fall in the same shard without having to be added. Since normalization values can be derived by summing the counts of all $n$-grams with the same history, the prefix is not strictly speaking required for normalization, though, as described in Section 3.2, we do add them when 'completing' a model shard to canonical WFST n-gram format.

Sharding with individual $\mathrm{n}$-grams as the unit rather than working with the more complex WFST topologies does have its benefits, particularly when it comes to relatively easy balancing of shards. The primary benefit of using WFSTs in such a distributed setting lies in making use of WFST functionality, such as modeling with expected frequencies derived from word lattices (Kuznetsov et al., 2016). Additionally, sharding on the suffix of the history does allow for scaling to much longer n-gram histories, such as would arise in character language modeling. If we train a 15-gram character language model from standard English corpora, then a significant number of those n-grams will begin with the space character, so creating a shard from a two character prefix may lead to extremely unbalanced sharding. In contrast, intervals of histories allow for balance even in such an extreme setting.

\section{Summary and Future Directions}

We have presented methods for distributing the estimation of WFST-based n-gram language models. We presented a model sharding approach that allows for much of the model estimation to be carried out on shards independently. We presented some pipeline algorithms that yield models identical with what would be trained on a single processor, and provided some data on what the resulting sharding looks like in real processing scenarios. We intend to create a full open-source distributed setup that makes use of the building blocks outlined here. 


\section{References}

Cyril Allauzen and Michael Riley. 2013. Preinitialized composition for large-vocabulary speech recognition. In Proceedings of Interspeech, pages $666-670$.

Cyril Allauzen, Michael Riley, Johan Schalkwyk, Wojciech Skut, and Mehryar Mohri. 2007. OpenFst: A general and efficient weighted finite-state transducer library. In Implementation and Application of $\mathrm{Au}$ tomata, pages 11-23. Springer.

Cyril Allauzen, Michael Riley, and Johan Schalkwyk. 2009. A generalized composition algorithm for weighted finite-state transducers. In Proceedings of Interspeech, pages 1203-1206.

Thorsten Brants, Ashok C Popat, Peng Xu, Franz J Och, and Jeffrey Dean. 2007. Large language models in machine translation. In Proceedings of the Joint Conference on Empirical Methods in Natural Language Processing (EMNLP) and Computational Natural Language Learning (CoNLL).

Craig Chambers, Ashish Raniwala, Frances Perry, Stephen Adams, Robert R Henry, Robert Bradshaw, and Nathan Weizenbaum. 2010. Flumejava: easy, efficient data-parallel pipelines. In ACM Sigplan Notices, volume 45-6, pages 363-375.

Ciprian Chelba, Thorsten Brants, Will Neveitt, and Peng $\mathrm{Xu}$. 2010. Study on interaction between entropy pruning and Kneser-Ney smoothing. In Proceedings of Interspeech, pages 2422-2425.

Ciprian Chelba, Tomas Mikolov, Mike Schuster, Qi Ge, Thorsten Brants, Phillipp Koehn, and Tony Robinson. 2014. One billion word benchmark for measuring progress in statistical language modeling. In Proceedings of Interspeech, pages 2635-2639.

Jeffrey Dean and Sanjay Ghemawat. 2008. Mapreduce: simplified data processing on large clusters. Communications of the ACM, 51(1):107-113.

Kenneth Heafield. 2011. Kenlm: Faster and smaller language model queries. In Proceedings of the Sixth Workshop on Statistical Machine Translation, pages 187-197. Association for Computational Linguistics.

Slava M. Katz. 1987. Estimation of probabilities from sparse data for the language model component of a speech recognizer. IEEE Transactions on Acoustics, Speech and Signal Processing, 35(3):400-401.

Vitaly Kuznetsov, Hank Liao, Mehryar Mohri, Michael Riley, and Brian Roark. 2016. Learning n-gram language models from uncertain data. In Proceedings of Interspeech (to appear).

Mehryar Mohri, Fernando Pereira, and Michael Riley. 2002. Weighted finite-state transducers in speech recognition. Computer Speech \& Language, 16(1):69-88.
Hermann Ney, Ute Essen, and Reinhard Kneser. 1994. On structuring probabilistic dependences in stochastic language modeling. Computer Speech and Language, 8:1-38.

Brian Roark, Richard Sproat, Cyril Allauzen, Michael Riley, Jeffrey Sorensen, and Terry Tai. 2012. The OpenGrm open-source finite-state grammar software libraries. In Proceedings of the ACL 2012 System Demonstrations, pages 61-66.

Hasim Sak, Yun-hsuan Sung, Françoise Beaufays, and Cyril Allauzen. 2013. Written-domain language modeling for automatic speech recognition. In Proceedings of Interspeech, pages 675-679.

Jeffrey Sorensen and Cyril Allauzen. 2011. Unary data structures for language models. In Proceedings of Interspeech, pages 1425-1428.

Andreas Stolcke. 1998. Entropy-based pruning of backoff language models. In Proceedings of the DARPA Broadcast News Transcription and Understanding Workshop, pages 270-274.

Ian H. Witten and Timothy C. Bell. 1991. The zerofrequency problem: Estimating the probabilities of novel events in adaptive text compression. IEEE Transactions on Information Theory, 37(4):10851094. 